\title{
Publisher Correction: Bridging cultural sociology and cognitive psychology in three contemporary research programmes
}

Michèle Lamont, Laura Adler, Bo Yun Park and Xin Xiang

Correction to: Nature Human Behavior https://doi.org/10.1038/s41562-017-0242-y (2017); published online 20 November 2017.

Owing to a technical error, Bo Yun Park's affiliation was incorrect in the originally published HTML version of this Perspective and should have read: Department of Sociology, Harvard University, Cambridge, MA, USA. This has now been corrected. The PDF version is correct. 